\title{
THE PATHOGENICITY OF BACTEROIDES FRAGILIS AND RELATED SPECIES ESTIMATED BY INTRA- CUTANEOUS INFECTION IN THE GUINEA-PIG
}

\author{
J. P. MASKELL \\ Department of Medical Microbiology, London Hospital Medical College, Turner Street, \\ London E1 $2 A D$
}

\begin{abstract}
SUMmARY. The pathogenicity of 42 strains of Bacteroides of human origin was estimated by intracutaneous injection of bacterial suspensions into guinea-pig skin. Comparisons of living and heat-killed suspensions revealed that $B$. fragilis strains maintained themselves and possibly multiplied in the skin, whereas the lesions induced by non-fragilis strains appeared to be due mainly to toxicity. Measurement of skin pathogenicity in terms of the number of viable organisms in the inoculum that produced a lesion $10 \mathrm{~mm}$ in diameter showed that $B$. fragilis was, on average, 17 times as pathogenic as non-fragilis strains.

Skin tests of pathogens may be of value in the analysis of virulence factors of Bacteroides and possibly of other anaerobic organisms.
\end{abstract}

\section{INTRODUCTION}

Bacteroides species are numerically the major group of micro-organisms isolated from normal faeces, and have been implicated in bacteraemia and in abdominal, gynaecological, obstetric and other infections. The group previously classified as Bacteroides fragilis has received much attention because it represents the anaerobic species most often associated with infection (Leigh, 1974). Until recently, $B$. fragilis was divided into five subspecies: fragilis, ovatus, distasonis, thetaiotaomicron and vulgatus, but differences between the subspecies based on DNA homology were found to be large enough to warrant reclassification into species (Cato and Johnson, 1976). B. fragilis is only a minor component of normal faecal isolates, but is more often isolated during infection than the species representing a higher percentage of normal faecal isolates, such as B. vulgatus and B. thetaiotaomicron (Finegold, Attebery and Sutter, 1974; Jones and Fuchs, 1976).

In a rat model of intra-abdominal sepsis (Weinstein et al., 1974) used to investigate potential virulence factors in this group of organisms (Onderdonk et al., 1977), the capsular polysaccharide of $B$. fragilis was found to be a potent virulence factor, rarely found in the other closely related species. Reports of synergy between Bacteroides and other organisms have been made and confirmed by animal experiments (Altemeier, 1942; Hite, Locke and Hesseltine, 
1949; Hill, Osterhout and Pratt, 1974; Kelly, 1978), but the relative contribution that each member of the synergic pairs makes to the total pathogenic effect does not appear to have been investigated.

This paper describes the use of intracutaneous infections in the guinea-pig to compare the pathogenicity of five species of Bacteroides, previously classified as $B$. fragilis.

\section{MATERIALS AND METHODS}

Micro-organisms. The following reference strains were used: B. fragilis NCTC9343 (designated strain 1 in this investigation), B. distasonis ATCC8503 (strain 2), B. ovatus ATCC8483 (strain 3), B. vulgatus ATCC8482 (strain 4) and NCTC10583 (strain 7), and B. thetaiotaomicron ATCC8489 (strain 5) and NCTC10582 (strain 6). Other strains were isolated from clinical specimens such as pus and wound swabs (s-strains), or from faeces (f-strains) at The London Hospital clinical laboratories. Altogether, 42 strains were investigated, consisting of the seven reference strains, 22 clinical isolates and 13 strains from faeces.

Culture media. For primary isolation, maintenance and culture from experimental lesions, nutrient agar (Southern Group Laboratories, Hither Green Hospital, London SE13 6RU) containing $7 \%$ lysed defibrinated horse-blood was used. All plate cultures were incubated anaerobically in GasPak jars (BBL, supplied by Becton-Dickinson (UK) Ltd, York House, Empire Way, Wembley, Middlesex) at $37^{\circ} \mathrm{C}$ for $48 \mathrm{~h}$. Antibiotic susceptibility was estimated on Diagnostic Sensitivity Test Agar (Oxoid) enriched with 7\% (v/v) lysed defibrinated horse-blood. Bacterial suspensions for animal inoculation were grown in Robertson's Cooked Meat Medium (Southern Group Laboratories). Fermentation reactions were determined in cooked-meat medium supplemented with $20 \%$ (w/v) filter-sterilised solutions of the carbohydrates to give a final concentration of $1 \%$.

Isolation of Bacteroides. Samples from clinical specimens and faeces were cultured on freshly poured lysed blood-agar and incubated anaerobically for $48 \mathrm{~h}$ at $37^{\circ} \mathrm{C}$. Colonies resembling Bacteroides were tested for purity and identified as described below.

Characterisation of strains was initially by their sensitivity patterns to erythromycin (60- $\mu \mathrm{g}$ disks) rifampicin $(15 \mu \mathrm{g})$, colistin sulphate $(10 \mu \mathrm{g})$, penicillin $\mathrm{G}$ (2 units), kanamycin (1000 $\mu \mathrm{g})$ and vancomycin $(5 \mu \mathrm{g})$, as described by Sutter and Finegold (1971). Strains resistant to all but erythromycin and rifampicin were assumed to be of the $B$. fragilis group; those giving other patterns were omitted from the investigation. B. fragilis strain NCTC9343 was included with each series of sensitivity tests as a reference.

Catalase and indole production was tested for in 48-h cooked-meat cultures by the methods of Holdeman, Cato and Moore (1977).

Carbohydrate fermentation. The fermentation of glucose, rhamnose, trehalose, mannitol and arabinose was determined by the method of Duerden et al. (1976) without growth supplements because all strains grew well without them.

Storage of cultures. The fluid from 24-h cooked-meat cultures was decanted and stored at $-70^{\circ} \mathrm{C}$ until required.

Preparation of suspensions for animal inoculations. Growth from 24-h cultures in cookedmeat broth was spun down and resuspended in saline $(\mathrm{NaCl} 0.85 \% \mathrm{w} / \mathrm{v}$ in water) to one-tenth the original volume of medium. Viable counts of suspensions were made in triplicate by the method of Miles, Misra and Irwin (1938) within $2 \mathrm{~h}$ of preparation. All plates were incubated anaerobically for $48 \mathrm{~h}$ and mean colony counts were made. Suspensions of killed organisms were prepared by heating in a waterbath at $80^{\circ} \mathrm{C}$ for $30 \mathrm{~min}$ and sterility was checked by plating on blood agar.

Animals. Batches of three female Hartley-strain albino guinea-pigs weighing 400-600 g were used for testing each set of suspensions. In any one experiment the weight range did not exceed $50 \mathrm{~g}$. The hair was clipped over the dorsal skin and flanks before intracutaneous injection. The resulting lesions were measured $24 \mathrm{~h}$ later, after depilation of the skin with a barium-sulphide paste (Miles and Miles, 1952). 
Measurement of intracutaneous pathogenicity. Skin pathogenicity was estimated by the method of Miles, Miles and Burke (1957). Undiluted suspensions and 1 in 5 dilutions of live and of killed organisms in saline were injected intracutaneously in $0 \cdot 1-\mathrm{ml}$ volumes, through a $10-\mathrm{mm}$ gauge 5/10 needle, into the dorsal skin and flanks of animals. From 16 to 20 injections were given at approximately $2 \cdot 5-\mathrm{cm}$ intervals to each set of animals so that four or five strains could be tested in one experiment. Suspensions were numbered and injected according to a partially randomised plan so that when the lesions were being measured it was not easy to determine which suspension had induced them. Suspensions containing about $10^{8} \mathrm{cfu} / 0 \cdot 1 \mathrm{ml}$ usually produced lesions of $c .10 \mathrm{~mm}$ diameter after $24 \mathrm{~h}$. Estimates of mean lesion diameters resulting from each suspension were made from single lesions induced in each of three animals. Intracutaneous injection of sterile saline alone failed to produce any apparent effect on the skin of animals. Non-circular lesions were measured across the greatest and least diameters and the average taken.

Lesion diameters were plotted against the $\log _{10}$ dose of living or dead organisms injected. In previous experiments with a series of Bacteroides suspensions of graded concentration, lesion diameters were found to be roughly linear with respect to $\log _{10}$ dose within the range of concentrations used; subsequent experiments were therefore performed with only two concentrations.

The ability of strains to produce lesions was expressed as the number of organisms in $0 \cdot 1 \mathrm{ml}$ required to induce a mean lesion diameter of $10 \mathrm{~mm}$; this value was calculated for living and dead suspensions of all strains and termed ED10(L) and ED10(D) respectively (Miles et al., 1957). In some cases, especially with non-fragilis strains, lesions were less than $10 \mathrm{~mm}$ in diameter even with undiluted suspensions, and in these instances plots based on two suspension concentrations were extrapolated to the $10 \mathrm{~mm}$ level. The ED10 values were used to compare the skin pathogenicity of live organisms of the different strains and of the skin toxicity of dead organisms. The ratio ED10(D): $E D 10(\mathrm{~L})$ was estimated for each strain to give a potency ratio, which was assumed to indicate the extent to which a given strain persisted or multiplied in the skin. On the assumption that the inflammatory reaction produced by dead bacteria was due to bacterial toxicity, a lesion size with living bacteria much bigger than that with dead suggested that the strain had survived effectively or even multiplied during its residence in the skin, whereas little or no difference between lesions of dead and living organisms suggested that they were largely due to microbial toxicity, with little or no multiplication. Strains with low ED10(L) values were clearly of greater skin pathogenicity than those with high values, and strains with low ED10(D): ED10(L) potency ratios $(<1: 1)$ were less pathogenic than those with high values $(>1: 1)$.

Content of viable bacteria in skin lesions. Some of the lesions induced by the injection of undiluted viable suspensions of Bacteroides were sampled after 24 or $48 \mathrm{~h}$ to establish the approximate concentrations of bacteria across the diameter of lesions (Miles, Pillow and Khimji, 1976). After the measurement of lesions, the animals were killed by carbon-dioxide inhalation and the depilated skin cleaned with $75 \%$ (v/v) ethanol in water and allowed to dry. From the periphery to the centre, each lesion was sampled at $2 \mathrm{~mm}$ intervals from 5 - $\mathrm{mm}$ parallel cuts made across the lesion at each interval. Samples were taken by rubbing a loop along both walls of each cut and plating directly on blood agar. After anaerobic incubation for $48 \mathrm{~h}$, the growth was graded as: 8 (confluent), 4 (semiconfluent), 2 ( $>20$ colonies) or 1 ( $<20$ colonies), and mean growth values were calculated from lesions in each set of animals. In one experiment three sets of three animals were given identical inocula of Bacteroides. One set was killed immediately after injection and the lesions were sampled; the other two sets were killed for sampling after 24 and $48 \mathrm{~h}$ respectively.

\section{RESULTS}

\section{Pathogenicity and toxicity of strains}

The lesions produced by all strains of Bacteroides were essentially similar, easily measured, discrete, circular areas of mild induration without obvious 
necrosis. B. fragilis always produced larger lesions, often of $50 \%$ greater diameter, than similar inocula of non-fragilis strains. Lesions induced by undiluted suspensions of non-fragilis strains were rarely greater than $7 \mathrm{~mm}(\overline{\mathrm{x}}$ $6.1 \mathrm{~mm})$ in diameter, whereas lesions of $9-18.5 \mathrm{~mm}(\overline{\mathrm{x}}=11.8 \mathrm{~mm})$ were often associated with $B$. fragilis; there was no obvious difference in diameter between the lesions caused by clinical and those caused by faecal isolates of $B$. fragilis.

\section{Relative lesions sizes induced by live and killed suspensions}

The plots of lesion diameter against $\log _{10}$ dose for dead and for living suspensions of a given strain were approximately parallel, with mean slopes of $1.96(\mathrm{SE} \pm 0.032)$ for non-fragilis, and 3.33 (SE \pm 0.07$)$ for $B$. fragilis $(\mathrm{p}<0.01$ by $t$-test). ED10 values were determined from slopes for live and killed suspensions of each strain. ED10(L) values for $B$. fragilis and non-fragilis strains were significantly different $(\mathrm{p}<0.01)$, with mean values of 9.34 and 10.56 respectively. Plots for about $20 \%$ of strains were not parallel, those for the live organisms being steeper than those for the killed. In these instances, for estimation of ED10 the slope for the living suspension was taken as standard, and a line was drawn through the midpoint of the slope for the killed bacteria parallel to that for the living.

$B$. fragilis lesions were always smaller when killed suspensions were given; the mean diameter decreased by about $33 \%$. Killed non-fragilis strains, with one exception, produced larger lesions (mean diameter increased by $33 \%$ ) than the corresponding viable suspensions. Typical slopes for $B$. fragilis and nonfragilis strains are shown in fig. 1; the ED10 of $B$. fragilis strain 7 (A slopes) was increased approximately 100 -fold when killed; that of $B$. thetaiotaomicron strain 17 (B slopes) was decreased by about 16 -fold.

The potency ratios were calculated for each strain to provide values representative of skin pathogenicity (table I). A ratio of greater than 1:1 suggests persistence or multiplication of viable organisms in the skin. $B$. fragilis strains generally had ratios of greater than $1: 1(\overline{\mathrm{x}}=1 \cdot 11: 1)$ whereas ratios for non-fragilis strains were usually less than $1: 1(\bar{x}=0.96: 1)$ because lesion diameters due to the killed organisms were often bigger than those for the living. These ratios for $B$. fragilis and non-fragilis strains differed significantly $(\mathrm{p}<0.01)$.

\section{Enumeration of viable bacteria in the lesions}

Lesions were sampled to determine whether lesion diameter indicated the extent to which viable organisms were able to persist or multiply in the tissue. The mean concentrations of viable organisms at intervals across the lesions are shown in table II, and examples of individual experiments are shown in fig. 2.

$B$. fragilis strains were better able to survive, and possibly multiply, in guinea-pig skin than non-fragilis strains. All the central and $2 \mathrm{~mm}$ samples from 24-h B. fragilis lesions gave a growth index of 8 whereas most non-fragilis lesions gave indices of only 1 or 2 . Although inocula varied considerably, the 


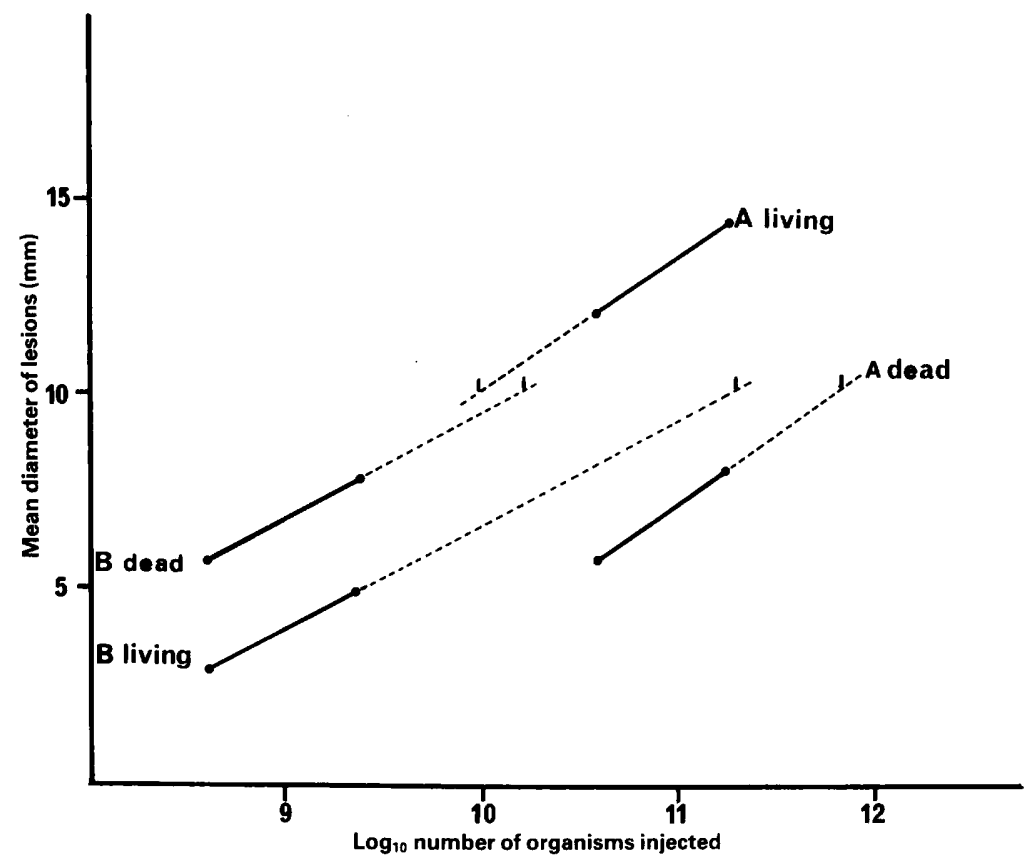

FIG. 1.-Examples of diameters of 24-h lesions induced by the injection of different numbers of living or dead suspensions of (A) B. fragilis (strain 7) and (B) B. thetaiotaomicron (strain 17). $\bullet-$ Observation, $----=$ extrapolation. Vertical lines at the $10 \mathrm{~mm}$ level indicate $\log _{10}$ ED 10.

numbers of viable $B$. fragilis strains 1 and 9 injected were less than the number of all four non-fragilis strains injected, yet samples taken after $24 \mathrm{~h}$ yielded substantially more growth than from the non-fragilis lesions (table II).

Compared with the growth from sites cultured immediately after injection, the growth indices of $B$. fragilis strains were not reduced at $24 \mathrm{~h}$ and reduced only moderately at $48 \mathrm{~h}$, but those of non-fragilis were considerably reduced at $24 \mathrm{~h}$ and markedly so at $48 \mathrm{~h}$ with no growth at distances beyond $4 \mathrm{~mm}$ of the lesion centre in both cases (fig. 2.). Lesion measurements produced by injecting live bacteria therefore appeared to reflect the underlying concentrations of viable organisms and suggest that $B$. fragilis is better able to persist or multiply in guinea-pig skin than non-fragilis strains, even when given at low concentrations.

\section{Discussion}

For a given inoculum, B. fragilis strains always produced lesions of larger diameter than non-fragilis strains and this property was associated with their greater ability to survive in the skin tissue. In all cases, $B$. fragilis produced smaller lesions when heat-killed, whereas non-fragilis lesions were either unaffected or larger. The latter effect may be due to the liberation of toxic material by heating. Lesions induced by non-fragilis strains appeared to result 
TABLE I

Pathogenicity of living and dead suspensions and the corresponding potency ratios of Bacteroides fragilis and non-fragilis strains of Bacteroides

\begin{tabular}{|c|c|c|c|c|c|c|c|c|}
\hline $\begin{array}{l}\text { B. fragilis } \\
\text { strain no. } \\
\text { (and source) }\end{array}$ & ED10(D) & ED10(L) & $\begin{array}{c}\text { Potency ratio } \\
\text { ED10(D): ED10(L) }\end{array}$ & $\begin{array}{l}\text { Non-fragilis } \\
\text { strain no. } \\
\text { (and source) }\end{array}$ & Species & ED10(D) & ED10(L) & $\begin{array}{c}\text { Potency ratio } \\
\text { ED10(D): ED10(L) }\end{array}$ \\
\hline $3(\mathrm{~s})$ & $13 \cdot 5$ & 9.9 & $1 \cdot 36$ & $45(f)$ & $\mathrm{v}$ & $12 \cdot 7$ & $11 \cdot 5$ & $1 \cdot 10$ \\
\hline $29(\mathrm{~s})$ & 13.0 & $9 \cdot 6$ & 1.35 & S4(r) & $\mathrm{v}$ & $10 \cdot 5$ & $10 \cdot 3$ & 1.02 \\
\hline $44(\mathrm{f})$ & $10 \cdot 5$ & $8 \cdot 6$ & $1 \cdot 22$ & $38(\mathrm{f})$ & $\mathrm{v}$ & $11 \cdot 4$ & $11 \cdot 1$ & 1.03 \\
\hline $7(\mathrm{~s})$ & 11.9 & 9.9 & $1 \cdot 20$ & S7(r) & $\mathrm{v}$ & $10 \cdot 2$ & $10 \cdot 1$ & 1.01 \\
\hline $34(\mathrm{~s})$ & $10 \cdot 1$ & $8 \cdot 6$ & 1.17 & $22(\mathrm{f})$ & $\mathrm{v}$ & $9 \cdot \overline{3}$ & $9 \cdot 6$ & 0.97 \\
\hline $23(\mathrm{f})$ & 9.9 & 8.7 & $1 \cdot 14$ & 6(s) & $\mathrm{v}$ & 9.9 & $12 \cdot 1$ & 0.82 \\
\hline $35(\mathrm{~s})$ & 9.9 & 8.9 & $1 \cdot 11$ & S2(r) & d & $10 \cdot 2$ & 9.3 & $1 \cdot 10$ \\
\hline $4(\mathrm{~s})$ & $10 \cdot 2$ & $9 \cdot 2$ & $1 \cdot 11$ & $28(\mathrm{~s})$ & d & $10 \cdot 3$ & $10 \cdot 3$ & 1.00 \\
\hline Si(r) & $11 \cdot 4$ & $10 \cdot 3$ & $1 \cdot 11$ & $32(\mathrm{f})$ & d & 11.7 & 11.8 & 0.99 \\
\hline $14(\mathrm{f})$ & 9.7 & 9.0 & 1.08 & $21(\mathrm{f})$ & d & $9 \cdot 2$ & 9.4 & 0.98 \\
\hline $10(\mathrm{~s})$ & 9.9 & $9 \cdot 2$ & 1.08 & $16(\mathrm{f})$ & d & $10 \cdot 2$ & $10 \cdot 9$ & 0.94 \\
\hline $13(\mathrm{~s})$ & $10 \cdot 6$ & $9 . \overline{9}$ & 1.07 & $31(\mathrm{f})$ & $\mathrm{t}$ & $9 \cdot \overline{2}$ & $9 \cdot 1$ & $1 \cdot 01$ \\
\hline $8(\mathrm{~s})$ & $10 \cdot 1$ & $9 \cdot 6$ & 1.05 & 26 (f) & $t$ & $10 \cdot 3$ & $10 \cdot 3$ & 1.00 \\
\hline $5(s)$ & $9 \cdot 2$ & $8 \cdot 8$ & 1.05 & S5(r) & $\mathrm{t}$ & $11 \cdot 7$ & $11 \cdot 8$ & 0.99 \\
\hline $9(\mathrm{~s})$ & $9 \cdot 0$ & $8 \cdot 6$ & 1.05 & $18(\mathrm{f})$ & $t$ & $9 \cdot 5$ & 9.8 & 0.97 \\
\hline $1(\mathrm{~s})$ & $9 \cdot 2$ & 9.0 & 1.02 & S6(r) & $\mathrm{t}$ & $10 \cdot 4$ & 10.9 & 0.95 \\
\hline $15(\mathrm{f})$ & $9 \cdot 8$ & $9 \cdot 6$ & 1.02 & $11(\mathrm{~s})$ & $\mathrm{t}$ & $9 \cdot 4$ & $10 \cdot 0$ & 0.94 \\
\hline \multirow[t]{7}{*}{$2(\mathrm{~s})$} & 10.8 & $10 \cdot 8$ & 1.00 & $19(\mathrm{f})$ & $\mathrm{t}$ & $10 \cdot 4$ & $11 \cdot 1$ & 0.94 \\
\hline & & & & $17(\mathrm{f})$ & $\mathrm{t}$ & $10 \cdot 1$ & $11 \cdot 3$ & 0.89 \\
\hline & & & & 24(f) & $\mathrm{t}$ & $10 \cdot 3$ & $12 \cdot 3$ & $0 \cdot 84$ \\
\hline & & & & S3(r) & 0 & $9 \cdot 6$ & $9 \cdot 8$ & 0.98 \\
\hline & & & & $25(\mathrm{f})$ & 0 & 9.5 & 9.9 & 0.96 \\
\hline & & & & $20(\mathrm{f})$ & o & $9 \cdot 0$ & $9 \cdot 4$ & 0.96 \\
\hline & & & & $12(\mathrm{~s})$ & o & $10 \cdot 5$ & $11 \cdot 4$ & 0.92 \\
\hline
\end{tabular}

$\mathrm{ED} 10=$ the number of organisms in $0.1 \mathrm{ml}$ required to induce a mean lesion diameter of $10 \mathrm{~mm} ;(\mathrm{L})=$ live organisms, (D) $=$ dead organisms. See Materials and methods.

$(\mathrm{s})=$ Clinical isolate, $(\mathrm{f})=$ faecal isolate, $(\mathrm{r})=$ reference strain.

$\mathrm{v}=B$. vulgatus, $\mathrm{d}=B$. distasonis, $\mathrm{t}=B$. thetaiotamicron, $\mathrm{o}=B$. ovatus. 
TABLE II

Mean growth of $B$. fragilis or non-fragilis strains from 24-h lesions

\begin{tabular}{|c|c|c|c|c|c|c|c|c|}
\hline \multirow[b]{2}{*}{ Species } & \multirow{2}{*}{$\begin{array}{c}\text { Strain } \\
\text { no. }\end{array}$} & \multirow{2}{*}{$\begin{array}{l}\log _{10} \\
\text { number of } \\
\text { organisms } \\
\text { injected }\end{array}$} & \multirow{2}{*}{$\begin{array}{l}\text { Mean } \\
\text { diameter } \\
\text { of lesions } \\
(\mathrm{mm})\end{array}$} & \multicolumn{5}{|c|}{$\begin{array}{l}\text { Growth* from samples } \\
\text { at indicated distance } \\
(\mathrm{mm}) \text { from centre }\end{array}$} \\
\hline & & & & 0 & 2 & 4 & 6 & 8 \\
\hline B. fragilis & $\left\{\begin{array}{r}1 \\
9 \\
14 \\
15\end{array}\right.$ & $\begin{array}{l}8 \cdot 96 \\
8 \cdot 39 \\
9 \cdot 60 \\
9 \cdot 84\end{array}$ & $\begin{array}{r}9 \cdot 8 \\
9 \cdot 6 \\
12 \cdot 8 \\
11 \cdot 0\end{array}$ & $\begin{array}{l}8 \\
8 \\
8 \\
8\end{array}$ & $\begin{array}{l}8 \\
8 \\
8 \\
8\end{array}$ & $\begin{array}{l}8 \\
4 \\
4 \\
4\end{array}$ & $\begin{array}{l}0 \\
1 \\
2 \\
4\end{array}$ & $\begin{array}{l}0 \\
0 \\
0 \\
1\end{array}$ \\
\hline non-fragilis & $\left\{\begin{array}{l}11 \\
12 \\
17 \\
19\end{array}\right.$ & $\begin{array}{l}9 \cdot 28 \\
9 \cdot 39 \\
9 \cdot 30 \\
9 \cdot 42\end{array}$ & $\begin{array}{l}6 \cdot 6 \\
5 \cdot 8 \\
6 \cdot 2 \\
5 \cdot 4\end{array}$ & $\begin{array}{l}8 \\
1 \\
2 \\
1\end{array}$ & $\begin{array}{l}2 \\
2 \\
2 \\
1\end{array}$ & $\begin{array}{l}1 \\
0 \\
1 \\
0\end{array}$ & $\begin{array}{l}0 \\
0 \\
0 \\
0\end{array}$ & $\begin{array}{l}0 \\
0 \\
0 \\
0\end{array}$ \\
\hline
\end{tabular}

* Mean from three lesions; $8=$ confluent growth, $4=$ semi-confluent, $2=>20$ colonies, $1=<20$ colonies.

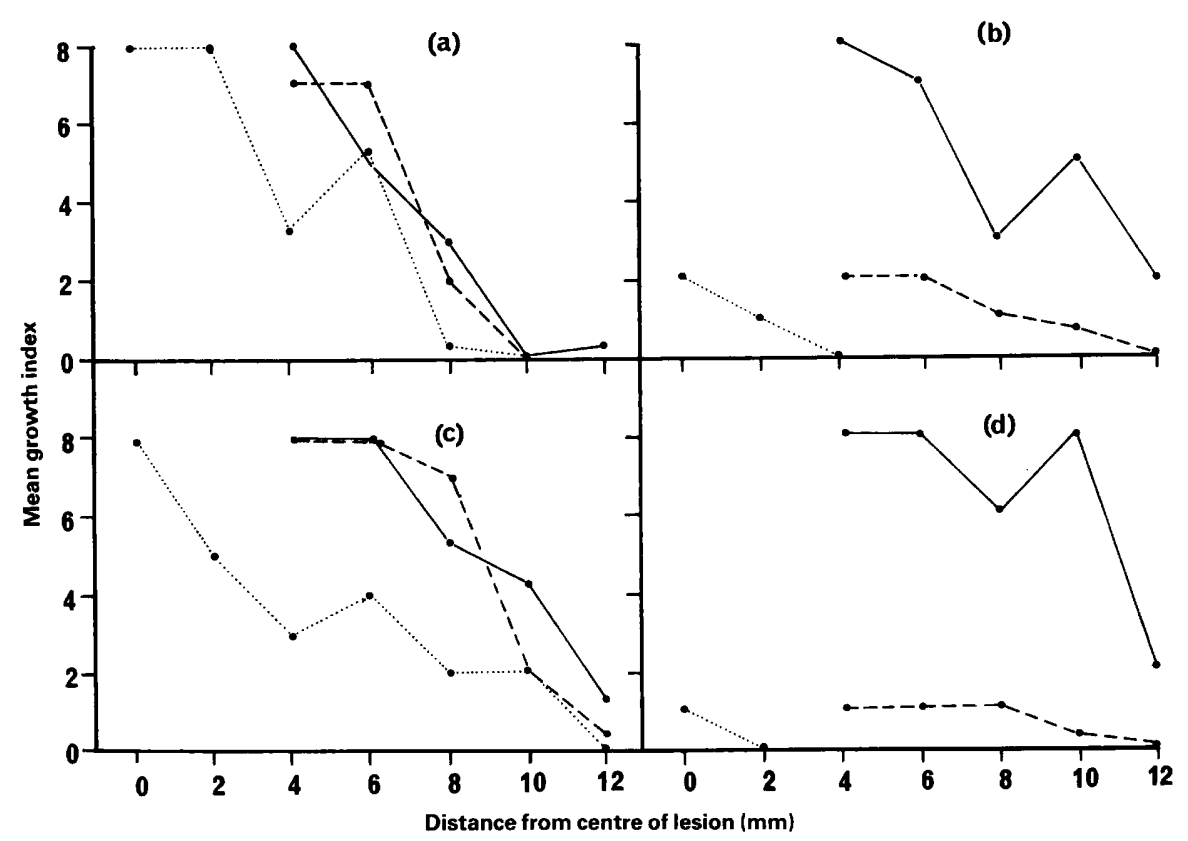

FIG. 2.-Examples of bacterial concentration profiles of $B$. fragilis strains 3 and 4 (a and $c$ ) and non-fragilis strains 16 and 24 (b and d) respectively, in lesions at various times after injection. $\bullet \bullet=0$-h samples, $\bullet-\bullet=24$-h samples, $\bullet \cdots=48$-h samples. For explanation of growth values, see footnote to table II. 
mainly from microbial toxicity with little multiplication of the inoculum. These estimates of the pathogenicity of Bacteroides, determined by the degree of persistence or multiplication in guinea-pig skin, confirm the findings of others that $\boldsymbol{B}$. fragilis is generally more pathogenic than the other closely related species of Bacteroides isolated from human material.

Reports concerning the ability of pure cultures of Bacteroides and other anaerobes to produce lesions or infections in experimental animals vary considerably. Altemeier (1942) and Hite et al., (1949) reported the production of infection by pure cultures of anaerobes, but their work has never been successfully repeated and it has been suggested (Dack, 1940, cited by Ingham, Sisson and Selkon, 1980) that the animals they used may have been fed on deficient diets. More recently, infections with $B$. fragilis have been produced in mice (Hill et al., 1974; Wilkins et al., 1977) with mucin or agar as a necessary component of the inoculum, and Kelly (1978) was able to produce deep wound infections in guinea-pig skin with pure cultures of $B$. fragilis.

Weinstein et al. (1974) described a two-stage infection culminating in abscess formation in rats given intraperitoneal implants of caecal contents, barium sulphate and Bacteroides. This model was later used by Onderdonk $e t$ $a l$. (1977) to investigate the relative pathogenicity of capsulated and non-capsulated strains of Bacteroides, the majority of the former being B. fragilis, which produced abscesses in $95 \%$ of animals tested; those given non-capsulated strains rarely developed abscesses. Of the potential virulence factors of $B$. fragilis tested, the highest incidence of abscess formation occurred with capsular polysaccharide which, in the absence of viable organisms, caused sterile abscesses. However, Babb and Cummins (1978) found that the presence of a capsule was not a unique characteristic of $B$. fragilis; indeed, all the species they tested, except $B$. distasonis, were capsulated to varying degrees.

Although the technique described in this paper was not intended to simulate natural infection, it has several advantages over the previously described methods. More tests can be performed on fewer animals, providing an index of pathogenicity within $24 \mathrm{~h}$. Whether pathogenicity as estimated in skin can be related to that in the common sites of $B$. fragilis infection, such as the gut, is as yet undetermined. However, the persistence or multiplication of organisms in living tissue, regardless of type, indicates an inherent ability to resist defence mechanisms of the host.

This guinea-pig model could be useful in the investigation of potential virulence factors because, unlike most other animal models, pure suspensions of Bacteroides spp. can be used to induce lesions, and the effects of potential virulence factors can be determined by estimating the enhancement of lesion diameter by the inclusion of such factors in the inoculum. Cellular components such as capsular polysaccharide, as well as extracellular enzymes such as proteases, lipases, nucleases and heparinases produced by Bacteroides spp. (Rudek and Haque, 1976), could be tested in this way. Factors enabling Bacteroides to inhibit phagocytosis (Ingham et al., 1977) could probably be assessed in this model. The importance of resistance to heat-labile serum components to the pathogenicity of Bacteroides, shown by B. fragilis but not 
the other species (Casciato et al., 1979), could be estimated by comparing lesions induced with and without a specific neutralising substance such as polyanethol sulphonate (Liquoid).

\section{REFERENCES}

ALtEMEIER, W. A. 1942. The pathogenicity of the bacteria of appendicitis peritonitis: experimental study. Surgery, 11, 374 .

BABB, J. L. AND Cummins, C. S. 1978. Encapsulation of Bacteroides species. Infect. Immun., 19, 1088.

Casciato, D. A., Rosenblatt, J. E., Bluestone, R., Goldberg, L. S. and Finegold, S. M. 1979. Susceptibility of isolates of Bacteroides to the bactericidal activity of normal human serum. J. infect. Dis., 140, 109.

CATo, E. P. AND Johnson, J. L. 1976. Reinstatement of species rank for Bacteroides fragilis, $B$. ovatus, $B$. distasonis, $B$. thetaiotaomicron and B. vulgatus: designation of neotype strains for Bacteroides fragilis (Veillon and Zuber) Castellani and Chalmers and Bacteroides thetaiotaomicron (Distaso) Castellani and Chalmers. Int. J. syst. Bact., 26, 230.

Duerden, B. I., Holbrook, W. P., Collee, J. G. AND WatT, B. 1976. The characterization of clinically important gram-negative anaerobic bacilli by conventional bacteriological tests. J. appl. Bact., 40, 163.

Finegold, S. M., AtTeber y, H. R. AND SutTer, V. L. 1974. Effect of diet on human fecal flora: comparison of Japanese and American diets. Am. J. clin. Nutr., 27, 1456.

Hill, G. B., Osterhout, S. AND Pratt, P. C. 1974. Liver abscess production by non-sporeforming anaerobic bacteria in a mouse model. Infect. Immun., 9, 599.

Hite, K. E., Locke, M. AND Hessel tine, H. C. 1949. Synergism in experimental infections with nonsporulating anaerobic bacteria. J. infect. Dis., 84, 1 .

Holdeman, L. V., Cato, E. P. AND Moore, W. E. C. 1977. Anaerobe laboratory manual, 4th ed., Virginia Polytechnic Institute and State University, Blacksburg, Virginia.

INGHAM, H. R., Sisson, P. R., AND SELKON, J. B. 1980. Current concepts of the pathogenetic mechanisms of non-sporing anaerobes:chemotherapeutic implications. J. antimicrob. Chemother., 6, 173.

Ingham, H. R., Sisson, P. R., Tharagonnet, D., Selkon, J. B. and Codd, A. A. 1977. Inhibition of phagocytosis in vitro by obligate anaerobes. Lancet, 2, 1252.

JONES, R. N. AND FUCHS, P. C. 1976. Identification and antimicrobial susceptibility of 250 Bacteroides fragilis subspecies tested by broth microdilution methods. Antimicrob. Agents Chemother., 9, 719.

Kelly, M. J. 1978. The quantitative and histological demonstration of pathogenic synergy between Escherichia coli and Bacteroides fragilis in guinea-pig wounds. J. med.Microbiol., $11,513$.

LeIGH, D. A. 1974. Clinical importance of infections due to Bacteroides fragilis and role of antibiotic therapy. Br. med. J., 3, 225.

MiLes, A. A. AND Miles, E. M. 1952. Vascular reactions to histamine, histamine-liberator and leukotaxine in the skin of guinea-pigs. J. Physiol., 118, 228.

Miles, A. A., MiLes, E. M. AND BuRKE, J. 1957. The value and duration of defence reactions of the skin to the primary lodgement of bacteria. Br. J. exp. Path., 38, 79.

Miles, A. A., MisRA, S. A. AND IRWIN, J. O. 1938. The estimation of the bactericidal power of the blood. J. Hyg., Camb., 38, 732.

Miles, A. A., Pillow, J. AND Khimji, P. L. 1976. The action of iron on local klebsiella infection of the skin of the guinea-pig and its relation to the decisive period in primary infective lesions. Br. J. exp. Path., 57, 217.

Onderdonk, A. B., Kasper, D. L., Cisneros, R. L. and Bartlett, J. G. 1977. The capsular polysaccharide of Bacteroides fragilis as a virulence factor:comparison of the pathogenic potential of encapsulated and unencapsulated strains. J. infect. Dis., 136, 82. 
Rudek, W. and Haque, R. U. 1976. Extracellular enzymes of the genus Bacteroides. J. clin. Microbiol., 4, 458.

SUTTER, V. L. AND FINEGOLD, S. M. 1971. Antibiotic disc susceptibility tests for rapid presumptive identification of Gram-negative anaerobic bacilli. Appl. Microbiol., 21, 13.

Weinstein, W. M., Onderdonk, A. B., Bartlett, J. G. AND Gorbach, S. L. 1974. Experimental intra-abdominal abscesses in rats:development of an experimental model. Infect. Immun., 10, 1250.

Wilkins, T. D., Walker, C. B., Nitzan, D. ANd Salyers, A. A. 1977. Experimental infections with anaerobic bacteria in mice. $J$. infect. Dis., 135, suppl. S 13. 